\title{
Déconstruire les paradigmes migratoires à travers les études sur les émigrations et les immigrations des femmes en Italie
}

Deconstructing Migration Paradigms through Studies on Female Emigration and Immigration in Italy

Deconstruyendo los paradigmas de la migración a través de estudios sobre la emigración y la inmigración femenina en Italia

\section{Adelina Miranda}

\section{(2) OpenEdition}

Journals

Édition électronique

URL : https://journals.openedition.org/remi/10279

DOI : $10.4000 /$ remi. 10279

ISSN : $1777-5418$

Traduction(s) :

Deconstructing Migration Paradigms through Studies on Emigration and Immigration of Women in Italy - URL : https://journals.openedition.org/remi/16885 [en]

Éditeur

Université de Poitiers

Édition imprimée

Date de publication : 1 avril 2018

Pagination : 173-194

ISBN : 979-10-90426-61-0

ISSN : 0765-0752

Référence électronique

Adelina Miranda, « Déconstruire les paradigmes migratoires à travers les études sur les émigrations et les immigrations des femmes en Italie ", Revue européenne des migrations internationales [En ligne], vol. 34 - $n^{\circ} 1$ | 2018, mis en ligne le 28 décembre 2019, consulté le 20 avril 2022. URL : http:// journals.openedition.org/remi/10279; DOI : https://doi.org/10.4000/remi.10279 


\section{Déconstruire les paradigmes migratoires à travers les études sur les émigrations et les immigrations des femmes en Italie}

\section{Adelina Miranda ${ }^{1}$}

Dans cette contribution, je propose d'examiner la mise en visibilité de la place des femmes dans les études sur les migrations en Italie. Pendant les années 1960, les immigrées commencent à arriver dans la Péninsule tandis que les émigrées continuent à partir à l'étranger. À l'époque, les premières ont été ignorées et les secondes ont été regardées comme n'accomplissant que leur devoir d'épouses, de mères ou de filles. Au cours des années 1970, les premiers travaux sur les femmes émigrées sont réalisés dans une perspective historique et anthropologique; à partir des années 1980, les études sur les femmes immigrées se développent en portant une attention particulière au travail du care. Depuis le début du XXle siècle, nous assistons à la complexification de cet espace épistémique. Si certain·e.s auteur.e.s utilisent le concept de genre comme une variable statistique qui fait référence à une représentation abstraite et universelle de la femme, étayant ainsi une vision androcentrée des migrations, d'autres ont adopté le prisme des rapports sociaux de sexe. Ces travaux étudient les imbrications des formes de domination dans une perspective intersectionnelle ${ }^{2}$ et, de ce fait, interrogent les paradigmes interprétatifs migratoires en général.

Pour mieux saisir ce cheminement, deux prémisses sont indispensables. La première est que, en Italie, les recherches sur les femmes migrantes ont d'abord été réalisées dans le champ des études sur les migrations et, plus tardivement, dans le champ des études sur le genre ${ }^{3}$; la deuxième est que les travaux sur la question genre/migration sont fortement influencés par la circulation internationale des idées, des théories et des chercheur.e.s. Le bilan partiel

1 Anthropologue, Professeure, MIGRINTER, Université de Poitiers/CNRS, MSHS, Bât. A5, 5 rue Théodore Lefebvre,TSA 21103, 86073 Poitiers cedex 9 ; adelina.miranda@univ-poitiers.fr

2 Ces études font référence à la perspective intersectionnelle développée depuis notamment les travaux de McCall (2005) et Crenshaw (1991).

3 Cette forme de circulation ouvre deux questions principales : comment s'est mis en place le dialogue entre ces champs d'études ? Et qu'est-ce que les études des migrations ont apporté aux études de genre et vice-versa ? Pour l'instant ces articulations n'ont pas été encore approfondies. 
que je présenterai s'insère dans ce contexte ${ }^{4}$. II rappelle que les études sur les émigrées et les immigrées ont démontré l'insuffisance des catégories interprétatives " classiques " en soulignant que les migrations féminines doivent tenir compte de thèmes typiquement féminins : la gestion de la santé, le care, l'éducation et l'élevage des enfants (Favaro et Tognetti Bordogna, 1991). Les deux traditions d'études ont de ce fait porté une attention fondamentale à la question du travail de reproduction, ce qui a permis d'appréhender I'enjeu des facteurs non économiques. Néanmoins, il faut remarquer que si les recherches réalisées sur les émigrées regardent souvent aux travaux menés sur les immigrées, il est plus rare qu'un détour historique soit opéré par celles-ci. Ce manque d'intérêt porte à opérer des comparaisons, souvent implicites, entre les migrantes du passé et celles actuelles, élaborées sur une série de généralisations antérieures dont " plus personne ne cherche à évaluer le bien-fondé " (Remaud, Schaub et Thireau, $2012: 14)^{5}$.

Dans cette contribution, je montrerai l'intérêt de mettre en relation les études sur les émigrées et sur les immigrées, à partir de trois questions. Quelle force explicative occupe encore aujourd'hui une vision androcentrée dans ces études ? Quelle place faut-il conférer à la variabilité avérée des situations migratoires féminines dans le passé et dans le présent ? Comment les études sur le genre peuvent contribuer à développer une vision critique des migrations ? Mon hypothèse est que questionner la césure, qui est souvent opérée entre les figures de l'émigrée et de l'immigrée, remet en cause l'usage de catégories interprétatives antinomiques hiérarchisantes. Intégrer dans nos réflexions I'hétérogénéité des situations migratoires féminines déplace notre attention du " pourquoi " au " comment " s'organisent les migrations et sur les articulations qui se créent entre le transnational, le national et le local tout en tenant compte de la force structurante des rapports de domination. Une étude conjointe des émigrations et des immigrations des femmes peut, de ce fait, constituer une base pour analyser les migrations comme étant des situations dynamiques et historicisées.

\section{À la recherche de la spécificité des femmes émigrées}

Comme le montre le bilan bibliographique dressé par Tirabassi (2015), à partir de la moitié des années 1970, les mondes de la recherche, académique et politique, commencent à s'intéresser aux femmes italiennes et à leurs descendantes vivant à l'étranger ${ }^{6}$. Ces enquêtes sur la " partie cachée " de l'émigration

4 J'opérerai une sélection de l'ample littérature qui, par ailleurs, est de moins en moins produite dans le contexte académique italien et en langue italienne.

5 Ce comparatisme implicite établit des continuités et des ruptures qui mettent sur le même plan les femmes italiennes d'antan et les femmes qui arrivent aujourd'hui en Italie dans le cadre du regroupement familial ou encore qui établissent des divergences entre les émigrées parties au cours des années 1960-1970 et celles qui partent depuis les années 2000 (Campani, 2000).

6 Déjà au début du XXe siècle, nous retrouvons des traces de l'attention portée aux femmes à travers une clef de lecture familiale ou victimiste (De Calboli, 1902), mais c'est notamment pendant les années 1970 que l'intérêt pour les femmes émigrées se développe tant dans le monde académique que politique. Voir à ce sujet : Charlot (1979) ; Yans-McLaughlin (1977); Knowles (1978); Caroli, Harney et Tomasi (1978) ; Mormino et Pozzetta (1983) ; Schiavo (1984) ; Chistolini (1986) ; Vecchio (1989). 
italienne ont constaté la spécificité des femmes tout en les libérant des valeurs de passivité et d'immobilité qui leur avaient été attribuées. Deux publications ont marqué cette tradition d'études. En 1983, la revue Studi Emigrazione publie un numéro intitulé La donna nei fenomeni migratori. Dans l'introduction de ce recueil d'articles issu d'un colloque qui avait eu lieu à Cagliari en 1982, Wihtol de Wenden (1983) évoque le poids d'une certaine vision féministe universaliste et évolutionniste sur le milieu de la recherche; elle souligne que les cultures d'origine des immigrées de la société méditerranée peuvent être à l'origine de formes de conflits, notamment intergénérationnels, et elle attire l'attention sur le fait que les migrations des femmes peuvent être accompagnées par une amélioration des conditions matérielles, mais également par des formes de déqualification professionnelle. En 1993, la revue Altreitalie publie huit articles sur les Italiennes émigrées en Argentine, Australie, Brésil, Canada et États-Unis. lacovetta (1993) rappelle que l'objectif de ce numéro était d'analyser les continuités et les changements, tant aux niveaux synchronique que diachronique, entre différents courants migratoires, mais que les contributions ont démontré la difficulté à opérer des comparaisons, étant donné la différente maturation de ce champ d'études selon les pays.

Ces études, alimentées par le débat étasunien des women's studies, ethnic studies et ethnic women's studies, ont été concomitantes avec le développement d'un regard novateur sur les femmes italiennes. La publication du volume Donne che vanno, donne che restano. Emigrazione e comportamenti femminili sous la direction de Corti (1990) a contribué à la reconnaissance de la mobilité des femmes comme étant un fait structurant le fonctionnement de la société italienne. Les recherches réalisées au cours de cette période à travers une approche historique et anthropologique permettent d'opérer quatre constats importants. Le premier constat est que le processus de féminisation des migrations s'enracine dans I'histoire du monde rural et que les transformations de celui-ci ne peuvent pas être expliquées sans tenir compte du rôle économique des femmes (qu'elles soient parties ou restées) et de la manière dont l'économie domestique s'est combinée avec les mouvements saisonniers ou définitifs tant des hommes que des femmes. Des nombreuses études de cas ont montré que l'autosuffisance économique assurée par les femmes a souvent permis le départ des hommes (Audenino, 1990 ; Merzario, 1996), surtout lorsqu'elle a été accompagnée du contrôle des naissances. Le deuxième constat est la coexistence de différentes formes de mobilités féminines. Les migrations temporaires, concernant notamment les jeunes filles, ont croisé les migrations saisonnières qui conduisaient régulièrement les femmes à parcourir des distances plus ou moins longues pour travailler dans le secteur reproductif comme nourrices ou lavandières ${ }^{7}$, ainsi que dans l'industrie textile ou dans l'agriculture. Ces mobilités pouvaient donc être un prolongement du travail domestique, mais elles répondaient surtout à la logique de la pluriactivité économique des femmes (Bianchi, 2001). Le troisième constat est l'importance occupée dans les systèmes migratoires par les femmes qui n'ont pas physiquement émigré. Regarder les migrations à partir de la place des femmes restées sur le lieu de départ a révélé 
la nécessité d'appréhender le choix individuel de partir ou rester sous l'angle des rapports sociaux de sexe à différentes échelles. Les femmes qui ne sont pas parties ont pris en charge les travaux dans les champs, même les plus lourds, en plus de l'élevage des enfants et de l'assistance des aînés ; elles ont également appris à administrer une économie qui n'était plus limitée à l'échelle locale (notamment à travers la gestion des remises) et leur propension à l'épargne a recouvert une fonction essentielle dans la réussite des projets migratoires. Le quatrième constat est que les migrations des hommes n'ont pas changé le statut des femmes restées sur le lieu de départ. En effet, le rôle de substitution des femmes dans les champs a été considéré comme étant temporaire et, pendant l'absence des hommes, il se produisait une sorte de "suspension de l'ordre de sexes " qui n'a pas effacé la subordination des femmes.

De leur côté, les recherches réalisées sur les lieux d'arrivée ont confirmé l'importance du rôle économique recouvert par les femmes migrantes et que leur insertion dans le monde du travail n'a pas été accompagnée par le prétendu " cycle d'intégration ". Les Italiennes qui partaient aux États-Unis au début du XXe siècle ont souvent trouvé un emploi dans les secteurs économiques informels et de la domesticité aussi bien que dans les industries ou dans le secteur de la confection au domicile (Tirabassi, 1993 ; Bianchi, 2001 ; Gabaccia et lacovetta, 2002). En France, les Italiennes ont été plus occupées dans le travail salarié au début du XXe siècle qu'au cours des années 1970 et leurs sorties et entrées sur le marché du travail se sont adaptées aux exigences économiques du groupe domestique et au déploiement, à travers le temps et les lieux, du projet migratoire familial (Miranda, 2001). De plus, les émigrées ont expérimenté une nouvelle gestion de la sphère reproductive et de sa " datità utilizzabile " ${ }^{8}, \mathrm{c}^{\prime}$ està-dire de la place que le monde domestique occupe en tant que lieu de sécurité primaire pour soi et pour les autres membres de la famille. Les émigrées ont dû apprendre à gérer les (parfois maigres) ressources économiques du foyer, à réorganiser leurs savoirs privés et intimes, à conférer une nouvelle valeur marchande à leur travail reproductif, parfois en monétarisant certaines tâches domestiques (Tirabassi, 1998 ; Gabaccia et lacovetta, 2002). Cette réorganisation de la domesticité leur a conféré un rôle central dans les projets migratoires, structurés par les temporalités tant individuelles que générationnelles ${ }^{9}$.

Les recherches sur l'émigration ont montré le lien étroit existant entre situations migratoires et structures de parentèle. Émigration et transmission du patrimoine se croisent avec les règles familiales et les logiques du mariage alimentant parfois la reproduction et parfois la manipulation de la tradition (Piselli, 1981 ; Ramella, 2001). C'est ainsi que des jeunes filles ont investi leur dot pour financier le départ de l'homme qu'elles venaient juste de marier ou ont mis en place des stratégies matrimoniales pour partir vers l'étranger ${ }^{10}$ ou des régions méridionales vers les campagnes du Piémont (Marchesano, 2010). L'attention

8 En se référant à la théorie de De Martino (2002), Signorelli (2000) a utilisé ce concept pour comprendre les dynamiques culturelles des mouvements de population.

9 Le départ et/ou la cohabitation des migrant·e.s, hommes et femmes appartenant à des générations différentes, a créé un nouvel espace d'interaction, d'interdépendance des actions individuelles.

10 Le film de Luigi Zampa Bello, onesto emigrato in Australia sposerebbe compaesana illibata (1972) met en scène d'une manière éclairante cette stratégie. 
portée aux dynamiques matrimoniales a démontré la spécificité des stratégies migratoires féminines et l'importance des négociations structurant la dispersion des parentés à travers les ajustements des logiques individuelles et familiales.

Les analyses sur la place occupée par la sphère domestique à l'intérieur des rapports de parenté ont permis la déconstruction du stéréotype de la femme émigrée italienne décrite comme une paysanne passive, emprisonnée dans les valeurs de la famille méditerranéenne de I'honneur et de la religiosité, qui se serait émancipée au travers de l'émigration, grâce à l'entrée dans le monde du travail productif. Comme l'ont bien souligné Tirabassi (1993) et Gabaccia (1998), ce stéréotype, élaboré sur les valeurs de la ségrégation spatiale féminine, a guidé les premières études sur les femmes italiennes aux États-Unis, mais il n'a jamais été corroboré par le vécu des femmes en situation migratoire qui souvent travaillaient déjà dans les campagnes italiennes.

De nombreuses études de cas confirment la variabilité du processus d'adaptation à la société et à la culture des pays d'installation. Les itinéraires individuels, notamment lorsqu'ils sont examinés dans et entre les parcours générationnels, mettent en exergue que des changements et des continuités ont remodelé la valeur attribuée à la famille et les rapports entre les ascendants, descendants et collatéraux. Les Italiennes arrivées en Australie, parfois après s'être mariées par procuration, ont transformé et adapté les traditions et, malgré le dur travail des champs, elles ont apporté des changements importants tant dans la sphère publique que privée de ce pays (Vasta, 1993). Aux États-Unis, certaines jeunes filles ont assumé des nouveaux rôles sociaux et elles ont remis en cause les relations de genre traditionnelles (Carneval, 2007) en se référant aux " groupes ethniques dominants "(Jones, 2007). Nombreuses, enfin, sont celles qui ont participé aux luttes sociales ${ }^{11}$ ou ont assumé des rôles transgressifs comme celui de devenir modèle pour les ateliers des artistes parisiens.

\section{L'arrivée des immigrées : I'effet structurant du travail du care}

En Italie, les questionnements sur les articulations existant entre l'appartenance de genre et l'immigration ont croisé la question de l'internationalisation des secteurs des services. À partir des années 1960, les premières migrantes arrivent en Italie en provenance des Philippines, de l'Érythrée, de la Somalie et du Cap-Vert, mais ce n'est que pendant les années 1990 qu'un certain intérêt scientifique s'est affirmé (Vicarelli, 1994). Ce manque d'attention pour les femmes immigrées résulte d'une vision androcentrique dominante dans les sciences sociales et du fait qu'à l'époque l'Italie n'est pas considérée comme un pays d'immigration, mais doit également être mise en relation avec le type d'insertion professionnelle des immigrés : dès le départ, les recherches ont fait état d'un pourcentage élevé de femmes travaillant dans le secteur domestique. Les nombreuses recherches réalisées sur les badanti (assistantes de vie) et les collaboratrici domestiche (femmes de ménage) ont relié l'arrivée des immigrées aux tendances économiques néo-libérales générées par des secteurs d'emploi féminin, toujours plus flexibles et précaires. La présence des migrantes a

11 À ce propos, voir les contributions de Gabaccia et lacovetta (2002, partie 3). 
été corrélée à l'émergence d'un nouveau modèle migratoire méditerranéen (Pugliese, 2012), qui faisait état d'un modèle bourgeois relevant de la demande de la domestique à temps complet.

Ces études ont toutefois laissé dans l'ombre tout un pan de la réalité migratoire féminine, comme la place des femmes entrepreneures, ou encore les continuités existant entre situations migratoires et situations prostitutionnelles. Et, surtout, elles ont ignoré les études sur les femmes italiennes. Pendant les années 1990, les recherches sur les conditions socio-économiques des Italiennes ont attiré l'attention sur leur faible taux d'insertion sur le marché du travail et sur le fait que leurs formes d'identification avec le rôle domestique étaient fortement en crise (Groppi, 1996 ; D'Amelia, 1997). Toutefois, les chemins vers I'émancipation n'étant pas linéaires, les changements se sont organisés sous forme de syncrétismes combinant éléments traditionnels et nouveaux. En Italie, les taux de chômage élevés des femmes et le refus du travail domestique ont coexisté avec une forte valorisation du rôle maternel et de la sphère de la consommation (D’Amelia, 1997 ; Siebert, 1999 ; Oppo, Piccone et Signorelli, 2000).

Cette réalité contredit l'idée que l'arrivée des étrangères doit être mise en relation avec l'entrée des Italiennes dans le monde du travail extradomestique, confirmant la fonctionnalité des flux d'immigration par rapport à l'émancipation des femmes autochtones. En effet, l'immigration féminine en Italie interroge plutôt ladite " crise du patriarcat " et son analyse rejoint la question posée par Signorelli (2011 : 38) à savoir est-ce que " les femmes ne sont-elles pas entraînées dans un processus qui attribue de nouvelles fonctions et des significations diverses aux formes de domination et de soumission déjà connues ".

Les études qui ont porté leur regard en même temps sur les femmes italiennes et immigrées ont montré que les rapports qui s'instaurent entre étrangères et Italiennes se construisent dans l'espace domestique, à l'intérieur d'une relation de genre qui crée une situation subalterne entre femmes appartenant à des cultures différentes (Miranda, 2002). Andall (2000) a mis en exergue que l'expérience migratoire des femmes cap-verdiennes, éthiopiennes, érythréennes et somaliennes arrivées en Italie entre les années 1970 à 1990 est liée au fait que les Italiennes, qui conservent entièrement leur rôle domestique, essayent de décharger une partie de leur travail sur les étrangères, ce qui permet de garder un certain équilibre social, notamment entre les sexes. En effet, l'arrivée et l'installation des immigrées ne découlent pas directement de la condition d'émancipation économique des Italiennes, mais de la constitution d'un marché du travail domestique englobant les rapports sociaux de sexe. En conséquence, le rôle économique recouvert par les immigrées ne peut pas être étudié en termes de "substitution " (comme l'on a fait pour les migrants travaillant dans les industries) ; il nécessite la révision des paradigmes interprétatifs androcentrés. Le fonctionnement du marché du travail reproductif dépasse les théories classiques de la demande et de l'offre ou encore des coûts et des bénéfices ; au croisement tant des systèmes migratoires que des formes de ségrégation du marché du travail, il est structuré par les effets des réseaux d'appartenance et par les itinéraires migratoires individuels (Catanzaro et Colombo, 2009).

Les études réalisées sur les femmes immigrées ont également mis au premier plan l'enchevêtrement existant entre motivations et causes dans les 
parcours migratoires. Toutefois, dans la plupart des cas, elles ont adopté une vision occidentalo-centrée. Ces recherches ont souligné comment les temporalités migratoires individuelles féminines sont reliées aux politiques migratoires ${ }^{12}$ mais, surtout, elles ont interprété l'expérience migratoire féminine comme étant différente de l'expérience masculine, liée à l'émergence de nouvelles aspirations, structurée par la recherche de l'autonomie et d'une réalisation personnelle et professionnelle qui seraient refusées aux femmes dans leurs sociétés de départ. La volonté d'échapper aux violences des hommes, à la répudiation, au mariage forcé a été évoquée comme étant des facteurs décisifs pour appréhender cette " culture du genre moderne " qui guiderait les immigrées vers des horizons plus égalitaires en terme de rapports sociaux de sexe. Cette lecture des migrations, qui établit que le choix opéré par les femmes est déterminé par une logique non économique, met en avant leur rôle culturel. Les migrantes sont habituellement décrites comme étant les " médiatrices entre les cultures" (Favaro et Tognetti Bordogna, 1991), les « vraies protagonistes d'une adaptation silencieuse et lente " (Mariti, 2003), comme "l'élément qui régule le processus d'intégration de la communauté " (Raffaele, 1992) ou encore comme celles qui contribueraient à la " création d'une société multiethnique " (Caritas/Migrantes, 2003). Dans cette perspective d'étude, l'appartenance de sexe se réduit à une sorte de variable qui prédispose, guide et détermine les comportements et attitudes migratoires des femmes.

"La femme immigrée " est ainsi transformée en un indicateur culturel qui informe - à travers les taux de scolarisation et de fécondité, l'insertion dans le monde du travail - du niveau d'émancipation du groupe social d'origine. Vue comme une " médiatrice " entre les cultures et les générations, le rôle de la femme migrante est inscrit dans les processus d'intégration à l'intérieur de la société d'arrivée. Cette vision essentialise l'appartenance culturelle qui, souvent réduite à la dimension religieuse, est évoquée comme l'élément explicatif des diversités entre femmes appartenant à des groupes nationaux différents ${ }^{13}$. La référence à une normativité "féminine " abstraite et universelle se constitue ainsi comme le support d'une pensée scientifique conformiste. Cette vision utilise l'appartenance de genre, établit dissimilitudes et similitudes entre femmes en provenance d'horizons culturels et économiques différents, et s'articule sur une opposition tradition/modernité = asservissement/émancipation des femmes (Miranda, 2008). Dans ce contexte, le concept de genre perd la force épistémologique qui a caractérisé les prémices de son usage pour, au contraire, soutenir une vision assimilationniste et définir les diverses actions menées auprès des migrantes par des institutions et des organismes qui souvent continuent à utiliser une vision androcentrée.

12 L'arrivée des migrantes a été favorisée par des régularisations qui dérogent au principe de fermeture des frontières nationales. Malgré la logique sécuritaire affichée en Italie, notamment depuis la loi Bossi-Fini (2001), en 2002 et en 2009, I'État a ouvert deux régularisations destinées notamment aux femmes employées comme badanti ou collaboratrici domestiche.

13 C'est ainsi des groupes nationaux profondément différents (les Polonaises et les Philippines par exemple) qui sont agrégés ; leurs parcours migratoires sont vus comme étant guidés par une même logique individuelle en opposition à celle des femmes en provenance du Maroc. 


\section{Une représentation persistante androcentrée}

L'actuelle présence d'étrangères en Italie et d'Italiennes à I'étranger soulève une série de questions à la fois sur les logiques du départ des femmes, sur l'incidence du taux de scolarisation, de la maternité et du travail sur leurs parcours migratoires. Ces interrogations essayent d'appréhender la question migratoire à travers une vision généralisante, qui fait référence à une catégorie abstraite de "femme migrante " laquelle ne reflète pas les situations variables tant dans l'espace que dans le temps et n'interroge pas la difficulté à trouver une ligne explicative unique pour éclairer les itinéraires des femmes.

Les recherches sur les différents groupes nationaux migrants ont confirmé cette hétérogénéité à différents niveaux. En premier lieu, elles ont démontré que même lorsque les femmes partent avec les hommes, leur rôle ne se réduit pas à celui de simples accompagnatrices. En deuxième lieu, elles ont constaté que la prise en compte de la religion comme variable indépendante occulte les enjeux liés à I'appartenance sociale, au statut matrimonial, au nombre d'enfants, à l'âge (Schmidt et Saint-Blancat, 1998 ; Macioti, 2000 ; Campani, 2000 ; Giacalone, 2002). En troisième lieu, un regard sur la longue durée a permis de mieux saisir les variations des dynamiques migratoires dans et entre les groupes (Vlase, 2006 ; Perra et Pilati, 2008 ; Pagnotta, 2012). Ainsi, les recherches sur les Italiennes en Suisse, en France, en Australie, en Argentine ont brossé un tableau qui restitue la complexité des situations, selon les échelles d'analyse adoptées (individuelle, familiale, collective) ${ }^{14}$. Ce cadre d'étude a par ailleurs été complexifié par la reprise des migrations italiennes internes et internationales qui ont souligné l'émergence de nouveaux modèles qui intéressent notamment des femmes célibataires avec un niveau d'études supérieur (Todisco et al., 2004 ; Pilato, 2011).

Bien que la mise en perspective des diverses situations migratoires féminines, dans le passé et dans le présent, montre une hétérogénéité des parcours et de l'expérience migratoire, la plupart des recherches continuent à utiliser une série d'indicateurs statistiques découlant $d^{\prime}$ 'une certaine vision androcentrée, comme le montre l'usage du sexe ratio. Celui-ci met en relation la participation des femmes aux flux migratoires avec celle des hommes sans tenir compte de l'action structurante des logiques de genre dans les stratifications sociales, de l'importance du rôle des femmes qui restent sur le lieu d'origine et de la présence significative des femmes dans les flux migratoires du passé.

Selon les inscriptions à l'AIRE (le registre des Italiens résidant à l'étranger) en 2014 , le nombre de femmes vivant à l'étranger possédant la nationalité italienne était de 2312 309, c'est-à-dire le $48 \%$ des 4811163 Italien·ne.s. Les "Italiennes de passeport " sont installées notamment en Argentine, en Allemagne, en Suisse, en France et au Brésil (Fondazione Migrantes, 2016) et elles se répartissent différemment selon les régions d'origine et les temporalités des migrations. Le taux de participation des femmes aux émigrations est donc

14 La littérature est désormais abondante. Voir parmi d'autres, Allemann-Ghionda et Meyer-Sabino (1992) ; Marengo (1993) ; lacovetta (1993) ; Vasta (1993) ; Niccolai (2004) ; Fibbi, Bolzman et Vial (1999); Gandolfo (1990) ; Grandi (2001); Bruno (2009) ; Arru et Ramella (2008); Rosa (2013) ; Borruso (2017). 
variable, mais il continue à être vu comme étant capable d'informer sur les typologies migratoires masculines ${ }^{15}$. Et cela malgré les recherches qualitatives qui soulignent que cet indicateur cache des réalités complexes et que, pour saisir la place occupée par les femmes dans les chaînes migratoires, il faut examiner les contextes économiques tant locaux qu'internationaux, le fonctionnement de la famille et du couple, ainsi que les politiques migratoires des pays de départ et d'arrivée.

Par exemple, selon les recensements français, les Italiennes représentaient plus du $40 \%$ en 1901 et $56 \%$ en 1975. Ces données confirment que la présence des femmes est liée au projet de stabilisation de la famille, mais une analyse plus approfondie montre que le départ des femmes n'a pas toujours été un " rendez-vous postdaté " avec les hommes. En effet, s'il est vrai que la présence féminine italienne est devenue plus visible avec l'arrivée définitive de toute la famille, de nombreux réseaux migratoires étaient composés exclusivement de femmes qui partaient comme ouvrières (Corazza, 1995) et surtout comme domestiques, nourrices ou bonnes à tout faire ${ }^{16}$. Par ailleurs, la stabilisation des familles a croisé les parcours des hommes qui continuaient à pratiquer des allers-retours. Des courants migratoires anciens (notamment en provenance du nord de I'Italie) se stabilisaient en se superposant aux nouveaux (notamment en provenance du sud du pays) où les hommes continuaient à faire les allersretours (Miranda, 2001 ; Sirna, 2005).

S'agissant des immigrations, les recherches ont d'emblée souligné un taux de présence élevé des femmes. Ce constat a été confirmé en 2015 : les femmes étrangères étaient presque aussi nombreuses que les hommes (49\% des 5026153 étrangers) (IDOS, 2016). Toutefois, leur présence se distribue différemment selon les nationalités. Les groupes marocain, albanais et roumain (parmi les plus nombreux en Italie) sont plus masculinisés que les groupes ukrainien, polonais, brésilien et moldave, qui sont plus féminisés. Une analyse diachronique montre en outre qu'au cours des trente dernières années nous avons assisté à une substitution et à une superposition entre et dans les flux migratoires féminins en Italie (Miranda, 2012). D'un côté, des groupes féminisés se sont masculinisés, comme dans le cas des Philippines. Au cours des années 1970, les immigrées qui sont arrivées en Italie étaient surtout célibataires et à la recherche d'une certaine autonomie ; pendant les années 1980, on observe l'arrivée de femmes mariées qui sont ensuite rejointes par les hommes. De l'autre côté, de nouveaux groupes nationaux composés notamment par des femmes se substituent à d'autres groupes migratoires féminisés. C'est le cas notamment des flux migratoires en provenance des ex-pays communistes. Ce processus de substitution entre les différents groupes féminins étrangers est la conséquence des politiques immigratoires italiennes qui cherchent à répondre aux besoins d'une société vieillissante. La gestion des décrets des flux migra-

15 Dans un passé récent, en partant du présupposé que les femmes suivent les hommes, on a calculé la quantité d'émigration définitive lorsque les femmes partaient et on a attribué le nombre de départs restant aux séjours temporaires pendulaires des hommes (De Clementi, 2011).

16 En 1901, plus de $90 \%$ des femmes italiennes étaient recensées comme « bonnes à tout faire ". Corti (1996) signale que déjà à la fin du XIXe siècle, environ 140000 Italiennes étaient employées de maison, bonnes de chambres ou domestiques dans les régions de la Savoie et de la Côte d'Azur. 
toires a permis le remplacement de la main d'ouvre féminine dans les secteurs du travail du care où le taux d'occupation parmi les femmes appartenant aux groupes plus féminisés resté très élevé. Cette condition, liée aux situations économique et politique des sociétés de départ et d'arrivée, a été utilisée pour élaborer une catégorisation qui oppose les "femmes seules " aux femmes " accompagnatrices ": les premières sont décrites comme étant des " actrices" de leur destin ; les secondes comme étant soumises au projet des leurs maris.

\section{Les articulations entre transnational et local}

L'adoption d'une approche transnationale a contribué à mieux saisir la place des femmes migrantes. Cette perspective examine les continuités entre lieux de départ et d'arrivée et dépasse la représentation de la migrante comme l'élément principal dans la transmission des traditions. En partant des études sur l'expérience maternelle transnationale, les recherches ont confirmé que, dans la plupart de cas, les migrantes gardent leurs responsabilités familiales et apprennent à agencer la distance physique et affective avec leurs enfants. Avec le départ, les formes de conciliation entre les responsabilités familiales et professionnelles changent : l'échelle spatiale se dilate et les liens s'agencent autour de temporalités différentes. Les (encore trop rares) études sur les migrantes de retour et sur les migrantes âgées confirment la variabilité des configurations familiales à travers le temps et que les projets migratoires individuels entrent en résonance avec différentes recompositions familiales. Les formes de regroupement aménagées par ces mères conduisent en effet à des typologies diverses de famille transnationale (Balsamo, 1997 ; Salih, 2000 ; Decimo, 2005 ; Ambrosini, 2009 ; Bonizzoni, 2007 ; Baggio, 2012).

Les recherches sur les émigrations réalisées dans cette même perspective ont démontré l'importance de l'approche comparative (Gabaccia, 1998) et, surtout, elles ont relativisé le poids du présent en corroborant le fait que les comportements transnationaux des migrantes ne sont pas nouveaux (Luconi et Varricchio, 2015). Les études réalisées à travers cette perspective ont mis en évidence que déjà dans le passé la constitution des champs migratoires internationaux a déterminé la plurilocalisation des membres des familles et que ce processus a généré de constants processus de délocalisation/relocalisation des relations affectives. L'approche transnationale a considéré les ajustements qui se sont créés entre ceux/celles qui partaient et ceux/celles qui restaient dépassant ainsi la vision cyclique de l'intégration (Gabaccia et lacovetta, 2002). Ces travaux ont de ce fait contribué à repenser les paradigmes interprétatifs dont nous disposons en soulignant qu'il faut considérer la complexité des conjonctions et des disjonctions qui se créent entre et au-delà des cadres nationaux, mais également entre les localismes.

Les études menées dans une perspective localisée sont très répandues dans le cadre italien. Elles sont une conséquence des politiques migratoires italiennes régionalisées, mais elles s'ancrent dans une tradition historique et anthropologique qui a porté son regard sur les dynamiques migratoires inscrites dans les réseaux des "compaesani ", des personnes originaires d'un même village d'origine. L'introduction de cette dernière échelle interroge les contextes de production du savoir et permet de dépasser les limites du nationalisme métho- 
dologique qui consiste à comprendre le monde social en prenant l'État-nation pour unité d'analyse.

L'attention consacrée aux petites échelles d'analyse montre que les migrantes jouent un rôle fondamental dans la recomposition des localités économiques et des ancrages affectifs, insérées dans les configurations transnationales. Ces femmes entretiennent les maisons familiales qui, au-delà des usages périodiques plus ou moins réguliers, recouvrent une valeur symbolique autour de laquelle se fixe la mémoire migratoire familiale. Une approche localisée permet en outre de connaitre quelle femme part, quelle expérience sociale et culturelle accompagne son parcours, mais également les contextes dans lesquels cette expérience se situe. De ce fait, elle offre un cadre d'analyse pour mieux saisir les logiques de genre et considérer en même temps les structures politiques, économiques et culturelles.

\section{L'enjeu des rapports sociaux de pouvoir}

Comme nous l'avons vu, les études sur les femmes immigrées en Italie ont pris en compte les similitudes et les différences existant entre les femmes migrantes et non migrantes ainsi qu'entre les femmes migrantes. Cette focale a contribué à déplacer le regard scientifique du profil migratoire individuel et/ou du groupe d'appartenance des migrantes aux articulations existant entre genre, ethnie et classe (Colombo, 2003 ; Nare, 2013). Les recherches menées à travers cette optique ont conduit à désencastrer les migrations des rapports de parenté et à déconstruire les essentialismes élaborés autour de la figure de la migrante. En déplaçant I'attention vers les structures sociales, politiques et culturelles, il apparaît que les conditions qui déterminent le départ et le devenir des migrantes varient selon les positions de force occupées dans l'espace migratoire. Cette perspective conduit vers l'étude des intersections entre le genre et les migrations, et vers l'étude de la force agissante des hiérarchies de pouvoir et de subalternité. Schmoll (2017), à partir de recherches menées sur les demandeuses d'asile, a montré que l'intersectionnalité permet de décrypter les perceptions, les pratiques et les comportements sociaux au regard des positions des migrantes dans la stratification sociale. Les migrations entrainent une reconfiguration des positions sociales de genre, de race et de classe qui résultent tant des actions individuelles que des effets de structures et des processus sociaux (comme la division sexuelle et raciale du travail) sur les migrantes. De ce fait, les femmes s'accommodent des hiérarchies existant, parfois elles les déjouent en situant les relations dans un jeu d'échelles réversible qui brouillent frontières et appartenances; ainsi, tandis que certaines peuvent œuvrer pour la reproduction de hiérarchies traditionnelles, d'autres peuvent provoquer des ruptures.

En même temps, la perspective intersectionnelle a mis en relief les articulations existant entre racisme ${ }^{17}$, sexisme et différentes formes d'oppression des femmes immigrées et a offert l'occasion aux féministes italiennes de resituer leur action. Après de longues années pendant lesquelles les associa-

17 Désormais, depuis les années 1980, le discours raciste s'est de plus en plus banalisé. Comme I'a bien remarqué Tabet (1997), I'arrivée des migrant·e·s a fait sortir I'Italie de la négation de son passée négationniste. 
tions des immigrées et des Italiennes se sont ignorées (Campani, 2011), nous assistons à l'émergence d'un mouvement qui interroge les interactions entre femmes en termes de race, d'ethnicité et de classe dans ses formes globales (Pojmann, 2006). Dans cette optique, les espaces de recherche prennent un sens politique. Ce positionnement est revendiqué par un certain nombre de jeunes chercheures ${ }^{18}$ qui soulignent que les discours sexualisés et genderisés sont essentiels dans la construction des processus d'ethnicisation; mieux, ils sont le support d'une politique qui utilise les migrations comme une stratégie de pouvoir (Bonfiglioli, 2012), car pour interpréter l'expérience migratoire, il ne $s^{\prime}$ agit pas de saisir seulement les difficultés vécues au niveau individuel, mais de comprendre comment lors des processus d'intégration opère la "color of skin " (Curti, 2007).

La critique de la pensée occidentale élaborée sur l'androcentrisme, l'hétérosexualité et l'ethnocentrisme genré passe à travers l'étude des articulations existant entre colonisations et migrations. L'article de Le Houérou publié dans ce dossier permet de comprendre comment le retour sur des sources historiques à travers une optique de genre dévoile des perspectives d'analyse inédites. La relecture de l'expérience coloniale est d'autant plus importante, car, comme le souligne Marchetti (2014), elle structure l'expérience migratoire des femmes, comme le montre le cas des Érythréennes. La situation migratoire reproduit les mécanismes de construction d'altérité dans l'intimité et crée la figure de la " postcolonial migrant domestic worker ". Le modèle actuel de la servitude est calqué sur celui de l'époque coloniale et, de ce fait, dans la construction des frontières entre migrantes et non migrantes, les rapports de domination coloniaux qui se réactivent passent tout d'abord par le corps des femmes et par leur utilisation dans le travail domestique. Une telle perspective reconnaît non seulement la valeur heuristique de l'étude de la pluralité des "points de vue " sur la réalité, mais, surtout, elle offre les bases pour comprendre les mondes différents dans lesquelles les sujets évoluent et donnent sens à leur positionnement dans les structures du pouvoir.

L'adoption d'une démarche féministe transnationale dans une perspective postcoloniale pose la question des différences et des inégalités pour développer un espace épistémique qui interroge l'universel afin de repenser les divisions et les hiérarchies de l'ordre social en termes d'intersectionnalité. En analysant I'" usage culturel " que les autochtones font de la figure des migrants qui traversent la Méditerranée à destination de I'Italie, j'ai pu remarquer qu'il est important d'adopter une démarche réflexive qui conduit à tenir compte tant de la manière de lire la présence de nouveaux migrants que de la manière qu'ont les Italiens de relier le phénomène migratoire à I'histoire nationale. La figure d'un homme arrivant seul, fuyant la guerre et les conflits (image contrastant avec la longue présence féminine étrangère en Italie) est devenue centrale dans cette élaboration des migrations qui fait désormais référence aux appartenances raciales. Les frontières sémantiques élaborées entre les Italiens et les " autres "

18 Voir le site du mouvement "Non una di meno " et notamment l'atelier "Femminismo Migrante " (https://nonunadimeno.wordpress.com/2017/02/08/tavolofemminismo-migrante/) et le cycle de conférences " Cartografie Subalterne: sguardi postcoloniali su confini, conflitti e traiettorie insorgenti " (https://www.youtube.com/ watch? $v=f 8 h c Z x B o z q s)$. 
évoquent l'imbrication genre/race élaborée autour de l'image du migrant homme/noir/sans-travail (Miranda, 2018).

En suivant ces travaux, il s'agit de poser la question des différences et des inégalités pour développer un espace épistémique qui contribue à l'émergence de nouveaux lieux de production des savoirs. En étudiant les actions menées par les membres des associations et des mouvements de Palerme et Vérone (Palermo Lesbicissima, Casa di Ramia, I'Italia sono anch'io, les Gaysprides), Alga (2015) a adopté une démarche ethnographique " postexotique " remettant en cause les formes de hiérarchisation des savoirs. Le nœud épistémologique d'un tel regard réflexif revendique, d'une part, une révision profonde des pratiques de terrain (afin de relativiser le point de vue scientifique en l'ouvrant à la confrontation avec d'autres points de vue) et, d'autre part, il interroge l'universel pour repenser les divisions et les hiérarchies de l'ordre social afin de contribuer à la redéfinition de la géopolitique de la connaissance et d'un espace épistémique mondialisé.

\section{Conclusions}

Les recherches sur les émigrées et les immigrées italiennes ont évolué dans le même espace épistémique, elles ont posé les mêmes questions et apporté des réponses complémentaires qui permettent d'avancer dans les études genremigrations à partir de trois constats. Le premier est que la complexification des flux migratoires est un processus en devenir, lié à une superposition constante des modalités migratoires genrées; le deuxième fait apparaître que les structures de pouvoir impliquent toutes les femmes, migrantes et non migrantes; le troisième souligne que les différences et les similitudes ne s'inscrivent pas dans des oppositions binaires (migrantes actuelles/du passé, migrante/non migrante). Ces travaux montrent l'insuffisance des modèles explicatifs classiques et mettent en évidence que l'étude des migrations doit prendre en compte d'une manière simultanée la mobilité et l'immobilité, les forces centrifuges et centripètes, les structures économiques et culturelles, les dimensions administratives et politiques.

En outre, une lecture conjointe des situations des immigrées et des émigrées défie les logiques évolutionnistes rendant difficile la formulation de généralisations ; elle montre l'insuffisance du concept de " femme migrante " et elle porte à considérer la diversité des situations migratoires comme étant structurée par la multidimensionnalité des interconnexions existant entre les phénomènes migratoires et les logiques de genre. De ce fait, ces études ouvrent une série de questions auxquelles seule une approche comparative peut donner une réponse. L'exercice réflexif croisé que j'ai proposé dans cette contribution vise à saisir la dimension genrée comme étant consubstantielle à la construction du savoir sur les migrations. En même temps, cette réflexivité théorique et méthodologique développée autour de la problématique des questions genre et migrations en Italie doit se poursuivre afin de contribuer à produire une critique des paradigmes interprétatifs des sciences sociales et à reconsidérer les rapports d'hégémonie et de subordination dans la production du savoir. 


\section{Références bibliographiques}

Alga Maria Livia (2015) "Ethnographie terrona » de sujets excentriques. Pratiques, narrations et représentations pour contrer le racisme et l'homophobie en Italie, Thèse de doctorat en anthropologie, Université Paris 8 Saint-Denis, 479 p.

Allemann-Ghionda Cristina e Meyer-Sabino Giovanna (1992) Donne italiane in Svizzera, Locarno, A. Dadò Editore, 295 p.

Ambrosini Maurizio (Ed.) (2009) Famiglie migranti, Mondi migranti, 1, pp. 35-131.

Andall Jacqueline (2000) Gender, Migration and Domestic Service. The politics of black women in Italy, Chippeham, Ashgate, $346 \mathrm{p}$.

Arru Angiolina (1996) Uomini e donne nel mercato del lavoro servile, in Angela Groppi Ed., Il lavoro delle donne, Bari, Editori Laterza, pp. 247-298.

Arru Angiolina e Ramella Franco (Eds.) (2008) Donne e uomini migranti. Storie e geografie fra breve e lunga distanza, Roma, Donzelli, 336 p.

Audenino Patrizia (1990) Le custodi della montagna: donne e migrazioni stagionali di una comunità alpina, Annali dell'Istituto Alcide Cervi, 12, pp. 265-287.

Badino Anna (2012) Strade in salita. Figli e figlie dell'immigrazione meridionale al Nord, Roma, Carocci Editori, 223 p.

Baggio Fabio (2012) Famiglie e bambini left-behind: il caso delle Filippine, Studi Emigrazione, 185, pp. 91-108.

Baldo Laura (1978) La doppia presenza, Inchiesta, 32, pp. 3-7.

Balsamo Franca (1997) Da una sponda all'altra del Mediterraneo. Donne immigrate e maternità, Torino, L'Harmattan Italia, $240 \mathrm{p}$.

Bevilacqua Piero, De Clementi Andreina e Emilio Franzina (Eds.) (2001) Storia dell'emigrazione italiana, Vol. 1 Partenze, Roma, Donzelli Editore, 847 p.

Bianchi Bruna (2001) Lavoro ed emigrazione femminile (1880-1915), in Piero Bevilacqua, Andreina De Clementi e Emilio Franzina Eds., Storia dell'emigrazione italiana, Vol. 1 Partenze, Roma, Donzelli Editore, pp. 257-274.

Bianchi Marina (1978) Oltre il " doppio lavoro ", Inchiesta, 32, pp. 7-11.

Bolzman Claudio et Fibbi Rosita (1993) Les immigrés face à la retraite : rester ou retourner?, Revue suisse d'Économie politique et de Statistique, 129, pp. 371-438.

Bonfiglioli Chiara (2012) Intersezioni di razzismo e sessismo nell'Italia contemporanea. Una critica dei recenti dibattiti femministi, in DWF, 87-88, pp. 64-76.

Bonizzoni Paola (2007) Famiglie transnazionali e ricongiunte: per un approfondimento delle famiglie migranti, in Mondi Migranti, 2, pp. 91-108.

Borruso Francesca (2017) In viaggio. Donne italiane migranti fra Otto e Novecento, Pedagogia Oggi, 15 (1), pp. 57-70.

Brunetta Giovanna (1983) Le donne e I'emigrazione: il caso Veneto, Studi Emigrazione, 70, pp. 154-163.

Bruno Oriana (2009) Le navi delle mogli: donne calabresi in Argentina, Altreitalie, 12 , pp. $61-84$. 
Campani Giovanna (2011) Les femmes immigrées dans une société bloquée : parcours individuels et organisations collectives en Italie, Cahiers du Genre, 51, pp. 49-67.

Campani Giovanna (2000) Genere, etnia e classe. Migrazioni al femminile tra esclusione e identità, Pisa, Edizioni ETS, 236 p.

Caponio Tiziana, Giordano Fedora, Manetti Beatrice e Ricaldone Luisa (Eds.) (2011) World Wide Women. Globalizzazione, Generi, Linguaggi. Volume 3, Torino, CIRSDe, [online]. URL: http://aperto.unito.it/bitstream/2318/804/1/9788890555 633.pdf

Caritas/Migrantes (2011) Immigrazione. Dossier statistico 2010, Roma, Edizioni Idos, $512 \mathrm{p}$.

Caritas/Migrantes (2003) Immigrazione. Dossier statistico 2002, Roma, Edizioni Idos, $512 \mathrm{p}$.

Carneval Nancy (2007) Le monde à l'envers : représentations des femmes dans le langage du théâtre comique italo-américain, in Natacha Lillo et Philippe Rygiel Dirs., Images et représentations du genre en migration (mondes atlantiques $X I X-X X e$ siècles), Paris, Publibook, $148 \mathrm{p}$.

Caroli Betty Boyd, Harney Robert and Tomasi Lydio (Eds.) (1978) The Italian Immigrant Woman in North America, Toronto, Ontario, Multicultural Historical Society, $386 \mathrm{p}$.

Catanzaro Raimondo e Colombo Asher (Eds.) (2009) Badanti \& Co. II lavoro domestico straniero in Italia, Bologna, II Mulino, 387 p.

Charlot Marie (1979) Visages de la femme immigrée italienne, Migrants Formation, 32-33, pp. 25-28.

Chistolini Sandra (1986) Donne Italoscozzesi. Tradizione e cambiamento, Roma, Centro Studi migrazione, $233 \mathrm{p}$.

Colombo Asher (2003) Razza, genere, classe. Le tre dimensioni del lavoro domestico in Italia, Polis, 17 (2), pp. 317-342.

Corazza Silvia (1995) Itinerari professionali femminili. Le setaiole di una comunità manifatturiera piemontese nella Francia medirionale, Recherches Régionales, 9, pp. 107-135.

Cortesi Gisella, Ghilardi Chiara e Maria Luisa Marengo (1999) Esperienze migratorie a confronto: donne italiane all'estero e donne straniere in Italia, in Carlo Brusa Ed., Atti del Convegno Immigrazione e multicultura nell'Italia di oggi, Milano, Franco Angeli, pp. 156-168.

Corti Paola (1996) L'émigration temporaire féminine piémontaise pendant la grande emigrazione : les images des sources, Cahiers de la Méditerranée, 52, pp. 163-172.

Corti Paola (Ed.) (1990a) Società rurale e ruoli femminili in Italia tra Ottocento e Novecento, Annali dell'Istituto Alcide Cervi, 12, 371 p.

Corti Paola (1990b) Donne che vanno, donne che restano. Emigrazione e comportamenti femminili, Annali dell'Istituto Alcide Cervi,12, pp. 213-235.

Corti Paola (1990c) Paesi d'emigranti. Mestieri, itinerari, identità collettive, Milano, Franco Angeli, 291 p. 
Crenshaw Kimberle (1991) Mapping the Margins: Intersectionality, Identity Politics, and Violence against Women of Color, Stanford Law Review, 43 (6), p. 1241-1299.

Curti Lidia (2007) Female Literature of Migration in Italy, Feminist Review, 87, pp. $60-75$.

D’Amelia Marina (Ed.) (1997) Storia della maternità, Bari, Editori Laterza, 410 p.

De Calboli Paolucci Roberto (1902) La tratta delle ragazze italiane, Nuova Antologia, [online]. URL: http://www.terzaclasse.it/documenti/trattadelledonne. htm

Decimo Francesca (2005) Quando emigrano le donne. Percorsi e reti femminili della mobilità transnazionale, Bologna, II Mulino, 236 p.

De Clementi Andreina (2011) Le donne nei flussi migratori italiani, in Adelina Miranda e Amalia Signorelli Eds., Pensare e ripensare le migrazioni, Milano, Sellerio, pp. 189-190.

De Martino Ernesto (2002) La fine del mondo, Torino, Einaudi, 727 p.

D'Ignazi Paola e Persi Rosella (2005) Migrazione femminile Discriminazione e integrazione tra teoria e indagine sul campo, Milano, Franco Angeli, 208 p.

Fabian Johannes (1990) Presence and Representation: The Other and Anthropological Writing, Critical Inquiry, 16 (4), pp. 753-772.

Favaro Graziella e Tognetti Bordogna Mara (1991) Donne dal Mondo. Strategie migratorie al femminile, Milano, Guerini, $248 \mathrm{p}$.

Fibbi Rosita, Bolzman Claudio et Vial Marie (1999) Italiennes et Espagnoles en Suisse à l'approche de l'âge de la retraite, Revue Européenne des Migrations Internationales, 15 (2), pp. 69-93

Fondazione Migrantes (2016) Rapporto Italiani nel Mondo 2016. Sintesi, Roma, Tav Editrice, 504 p.

Gabaccia Donna (1998) Le immigrate italiane in prospettiva comparata, in Altreitalie, 9, pp. 154-162.

Gabaccia Donna and lacovetta Franca (Eds.) (2002) Women, Gender, and Transnational Lives. Italian Workers of the World, Toronto, University of Toronto Press, $433 \mathrm{p}$.

Gandolfo Romolo (1990) Dall'alto Molise al centro di Buenos Aires: le donne agnonesi e la prima emigrazione transatlantica (1870-1900), Annali dell'Istituto Alcide Cervi, 12, pp. 325-351.

Giacalone Fiorella (2002) Marocchini tra due culture. Un'indagine etnografica sull'immigrazione, Milano, Franco Angeli, 240 p.

Ginatempo Nella (Ed.) (1992) Donne del Sud. Il prisma femminile sulla questione meridionale, Palermo, Gelka, $335 \mathrm{p}$.

Grandi Casimira (2001) L'emigrazione femminile italiana in Germania: i motivi di una scelta (1870-1914), Studi Emigrazioni, 142, pp. 346-374.

Groppi Angela (Ed.) (1996) I/ lavoro delle donne, Bari, Editori Laterza, 530 p. 
Guglielmo Jennifer (2010) Transnational Feminism's Radical Past: Lessons from Italian Immigrant Women Anarchists in Industrializing America, Journal of Women's History, 22 (1), pp. 10-33.

lacovetta Franca (1993) Scrivere le donne nella storia dell'immigrazione: il caso italo-canadese, Altreitalie, 9, pp. 5-24.

IDOS (2016) Dossier Statistico Immigrazione 2015, Roma, IDOS/UNAR, 467 p.

Jones Ginger (2007) De Bella à Belle : les femmes américaines originaires de I'Italie méridionale en Louisiana, in Natacha Lillo et Philippe Rygiel Dirs., Images et représentations du genre en migration (mondes atlantiques XIX-XXe siècles), Paris, Publibook, pp. 148-159.

Knowles Joan (1978) Maria: a migrant woman's story, Social Alternatives, 1 (2), pp. 57-59.

Luconi Stefano e Varricchio Mario (Eds.) (2015) Lontane da casa. Donne italiane e diaspora globale dall'inizio del Novecento ad oggi, Torino, Accademia University Press, $354 \mathrm{p}$.

Macioti Maria Immacolata (2000) La solitudine e il coraggio. Donne marocchine nella migrazione, Milano, Guerini e Associati, 190 p.

Marchesano Laura (2010) Alla ricerca di una moglie. Celibato rurale e migrazioni matrimoniali, Storicamente, 6, [online]. URL: http://www.storicamente.org/07_ dossier/famiglia/marchesano_immigrazione_matrimoniale.htm

Marchetti Sabrina (2014) Black Girl. Migrants Domestic Workers and Colonial Legacies, Leidu-Boston, Brill, 216 p.

Marengo Marina (1993) L'immigrée italienne sur le marché du travail suisse : le cas du canton de Vaud, Revue suisse d'économie politique et de statistique, 3, pp. 385-399.

Mariti Cristina (2003) Donna migrante. II tempo della solitudine e dell'attesa, Milano, Franco Angeli, 144 p.

McCall Leslie (2005) The Complexity of Intersectionality, Signs, 30 (3), pp. 17711800.

Merzario Raul (1996) Donne sole nelle valli e nelle montagne, in Groppi Angela Ed., Il lavoro delle donne, Bari, Editori Laterza, pp. 229-246.

Mignolo Walter (2001) Géopolitique de la connaissance, colonialité du pouvoir et différence coloniale, Multitudes, 6 (3), pp. 56-71.

Miranda Adelina (2018) Contrastes d'images. Les usages culturels de la figure des migrants arrivant en Italie par la Méditerranée, Revue Science and Video, 7, [en ligne]. URL : http://scienceandvideo.mmsh.univ-aix.fr/numeros/7/Pages/02. aspx

Miranda Adelina (2015) Sguardi incrociati sulle emigrazioni e le immigrazioni italiane, in Ornella De Rosa e Donato Verrastro Dirs., Studi di storia contemporanea: società, istituzioni, territori, Roma, libreriauniversitaria.it, pp. 219-239.

Miranda Adelina (2012) Per un approccio di genere allo studio delle migrazioni, in Caritas/Migrantes, Immigrazione. Dossier statistico 2011, Roma, Edizioni Idos, pp. 105-111. 
Miranda Adelina (2010) Lavoro riproduttivo, migrazioni e processi di alterizzazione femminili, in Luigi M. Lombardi Satriani Ed., RelativaMente. Nuovi territori scientifici e prospettive antropologiche, Roma, Armando Editore, pp. 215-222.

Miranda Adelina (2008) Migrare al femminile. Dinamiche culturali e appartenenza di genere in situazioni migratorie, Milano, McGraw-Hill, 198 p.

Miranda Adelina (2007) Le donne italiane in emigrazione tra passato e presente, in Fondazione Migrantes, Rapporto Italiani nel mondo 2007, Roma, Edizioni Idos, pp. 133-144.

Miranda Adelina (2002) Domestiche straniere e datrici di lavoro autoctone. Un incontro culturale asimmetrico, Studi Emigrazione, 148, pp. 859-879.

Miranda Adelina (2001) Les femmes et les entreprises italo-françaises dans la région parisienne, Migrations Société, 78, pp. 81-96.

Miranda Adelina e Signorelli Amalia (Eds.) (2011) Pensare e ripensare le migrazioni, Milano, Sellerio, $328 \mathrm{p}$.

Mormino Gary R. and Pozzetta George E. (1983) Immigrant Women in Tampa: The Italian Experience, 1890-1930, The Florida Historical Quarterly, 61 (3), pp. 296-312.

Nare Lena (2013) Migrancy, Gender and Social Class in Domestic Labour and Social Care in Italy: An Intersectional Analysis of Demand, in Journal of Ethnic and Migration Studies, 39 (4), pp. 601-623.

Niccolai Roberto (2004) «E tutti va in Francia, in Francia per lavorare ». La storia di una famiglia pistoiese emigrata in Francia, in Paola Foschi e Renzo Zagnoni Eds., Migranti dell'Appennino. Atti della Giornata di studio (Capugnano, 7 settembre 2002), Porretta Terme/Pistoia, Societa Pistoiese, pp. 189-203.

Oppo Anna, Piccone Stella Simonetta e Signorelli Amalia (Eds.) (2000) Maternità, identità, scelte. Percorsi dell'amancipazione femminile nel Mezzogiorno, Milano, Liguori, 269 p.

Pagnotta Chiara (2012) Attraverso lo stagno, Roma, CISU, 261 p.

Perra Sabrina e Pilati Katia (2008) Percorsi migratori femminili ed integrazione socioeconomica delle donne immigrate a Milano, Sociologia del Lavoro, 110, pp. 213-226.

Pilato Pamela (2011) Partire da sole: migrazioni femminili dalla Campania all'Emilia-Romagna, Sociologia del Lavoro, 121, pp. 152-167.

Piselli Fortunata (1981) Parentela ed emigrazione. Mutamenti e continuità in un comunià calabrese, Torino, Einaudi, 393 p.

Pojmann Wendy Ann (2006) Immigrant Women and Feminism in Italy, Burlington, VT, $188 \mathrm{p}$.

Pugliese Enrico (2012) II modello migratorio mediterraneo dell'immigrazione, in Adelina Miranda e Amalia Signorelli Eds., Pensare e ripensare le migrazioni, Milano, Sellerio, pp. 48-60.

Raffaele Giovanni (1992) Le immigrate extracomunitarie in Italia, Studi Emigrazione, 106, pp. 194-225. 
Ramella Franco (2001) Reti sociali, famiglie e strategie migratorie, in Piero Bevilacqua, Andreina De Clementi e Emilio Franzina Eds., Storia dell'emigrazione italiana, Vol. 1 Partenze, Roma, Donzelli Editore, pp. 143-160.

Remaud Olivier, Schaub Jean-Frédéric et Thireau Ișabelle (Dirs.) (2012) Pas de réflexivité sans comparaison, Paris, Éditions de l'École des hautes études en sciences sociales, pp. 13-20.

Rosa Silvia Giovanna (2013) Italiane d'Argentina. Storia e memorie di un secolo d'emigrazione al femminile (1860-1960), Torino, Ananke, 230 p.

Saillant Francine, Kilani Mondher et Graezer Bideau Florence (Dirs.) (2001) Manifeste de Lausanne. Pour une anthropologie non hégémonique, Montréal, Liber, 143 p.

Salih Ruba (2000) Identità, modelli di consumo e costruzione di sé tra il Marocco e I'Italia. Una critica di genere alla migrazione transnazionale, Afriche Orienti, 3, pp. 26-33.

Sarti Raffaella (2004) Noi che abbiamo visto un'altra città, abbiamo un'alra cultura. Servizio domestico, migrazioni e identità di genere in Italia: uno sguardo di lungo periodo, Polis. Ricerche e studi su società e politica in Italia, 18, pp. 17-46.

Schiavo Myrthia (1984) Italiane in Belgio. Le emigrate raccontano, Naples, Pironti, 228 p.

Schmidt di Friedberg Ottavia et Saint-Blancat Chantal (1998) L'immigration au féminin : les femmes marocaines en Italie du Nord. Une recherche en Vénétie, Studi Emigrazione, 131, pp. 483-498.

Schmoll Camille (2017) Spatialités de la migration féminine en Europe du Sud. Une approche par le genre, Rapport du dossier pour une Habilitation à Diriger les Recherches, Université de Poitiers.

Siebert Renate (1999) Cenerentola non abita più qui. Uno sguardo di donna sulla realtà meridionale, Torino, Roseberg e Sellier, $247 \mathrm{p}$.

Signorelli Amalia (2011) Genre : un concept désormais inutile, Journal des anthropologues, 124-125, pp. 25-48.

Signorelli Amalia (2006) Migrazioni e incontri etnografici, Palermo, Sellerio, $248 \mathrm{p}$.

Signorelli Amalia (2000) Le molteplici singolarità di un processo di massa. Alcune riflessioni sullo studio antropologico delle migrazioni, Etnoantropologia, 8-9, pp. 285-295.

Sirna Francesca (2005) Italiani in Francia: un'integrazione riuscita?, Studi Emigrazione, 160, pp. 786-805.

Tabet Paola (1997) Le pelle giusta, Torino, Einaudi, 208 p.

Tirabassi Maddalena (2015) Trent'anni di studi sulle migrazioni di genere in Italia, in Stefano Luconi e Mario Varricchio Eds., Lontane da casa. Donne italiane e diaspora globale dall'inizio del Novecento a oggi, Torino, Academia University Press, pp. 19-39, [online]. URL: https://books.openedition.org/aaccademia/891

Tirabassi Maddalena (1993) Italiane ed emigrate, Altretitalie, 9, pp. 5-23. 
Todisco Enrico, Cristaldi Flavia, Cariani Claudia e Tattolo Giovanna (2004) La skilled migration al femminile: il caso delle donne italiane in Svizzera, Studi Emigrazione, 156, pp. 831-867.

Vasta Ellie (1993) II cambiamento socioculturale: le donne italo-australiane e la seconda generazione, Altreitalie, 9, pp. 69-101.

Vecchio Diane C. (1989) Italian Women in Industry: The Shoeworkers of Endicott, New York, 1914-1935, Journal of American Ethnic History, 8 (2), pp. 60-86.

Vicarelli Giovanna (Ed.) (1994) Le mani invisibili. La vita e il lavoro delle donne immigrate, Roma, Ediesse, 248 p.

Vlase lonela (2006) Donne rumene migranti e lavoro domestico in Italia, Studi Emigrazione, 161, pp. 6-22.

Wihtol de Wenden Catherine (1983) Presentazione, Studi Emigrazione, 70, pp. 130-131.

Yans-McLaughlin Virginia (1977) Family and Community: Italian Immigrants in Buffalo, 1880-1930, Champaign, University of Illinois Press, 286 p.

Zampa Luigi (1972) Bello onesto emigrato Australia sposerebbe compaesana illibata, $115 \mathrm{~min}$. [online]. URL: https://www.google.fr/search?q=Bello,+onesto+ emigrato+in+Australia+sposerebbe+compaesana+illibata+(1972\&rlz=1C1GGRV enFR751FR751\&source=Inms\&tbm =vid\&sa=X\&ved=0ahUKEwiluoXvrJXeAhVQYUKHSR_Db0Q_AUIDygC\&biw $=1280 \& b i h=610$ 


\section{Adelina Miranda}

\section{Déconstruire les paradigmes migratoires à travers les études sur les émigrations et les immigrations des femmes en Italie}

Les recherches sur les émigrées et les immigrées italiennes ont évolué dans le même espace épistémique, elles ont posé les mêmes questions et apporté des réponses complémentaires qui permettent d'avancer dans les études genremigrations à partir de trois constats. Le premier est que la complexification des flux migratoires est un processus en devenir, lié à une superposition constante des modalités migratoires genrées ; le deuxième fait apparaître que les structures de pouvoir impliquent toutes les femmes, migrantes et non migrantes; le troisième souligne que les différences et les similitudes ne s'inscrivent pas dans des oppositions binaires (migrantes actuelles/du passé, migrante/non migrante). La réflexivité théorique et méthodologique développée autour de la problématique des questions genre et migrations en Italie permet de saisir la dimension genrée comme étant consubstantielle à la construction du savoir sur les migrations. Ces travaux montrent l'insuffisance des modèles explicatifs classiques et mettent en évidence que l'étude des migrations doit prendre en compte d'une manière simultanée la mobilité et l'immobilité, les forces centrifuges et centripètes, les structures économiques et culturelles, les dimensions administratives et politiques.

\section{Deconstructing Migration Paradigms through tudies on Female Emigration and Immigration in Italy}

Research on Italian emigrants and immigrants women has evolved in the same epistemic space; they have asked the same questions and provided complementary answers that allow progress in gender-migration studies around three observations. The first is that the complexity of migration flows is a developing process, linked to a constant superposition of gendered migration patterns; the second shows that power structures involve all women, both migrant and nonmigrant; the third emphasizes that differences and similarities are not binary opposites (current/past migrants, migrant/non-migrant). The theoretical and methodological reflexivity developed around the issue of gender and migration issues in Italy makes it possible to grasp the gender dimension as being consubstantial to the construction of knowledge on migration. This work shows the inadequacy of traditional explanatory models and highlights that the study of migration must simultaneously take into account mobility and immobility, centrifugal and centripetal forces, economic and cultural structures, administrative and political dimensions. 


\section{Deconstruyendo los paradigmas de la migración a través de estudios sobre la emigración y la inmigración femenina en Italia}

La investigación sobre las emigrantes e inmigrantes italianas ha evolucionado en el mismo espacio epistémico, ha hecho las mismas preguntas y ha proporcionado respuestas complementarias que permite avanzar en los estudios sobre la migración de género basados en tres observaciones. La primera es que la complejidad de los flujos migratorios es un proceso en desarrollo, vinculado a una superposición constante de patrones de migración de género; la segunda muestra que las estructuras de poder involucran a todas las mujeres, tanto migrantes como no migrantes; la tercera enfatiza que las diferencias y las similitudes no son binarias opuestas (migrantes actuales/pasados, migrantes/ no migrantes). La reflexividad teórica y metodológica desarrollada en torno a la cuestión del género y la migración en Italia permite entender la dimensión de género como consustancial a la construcción del conocimiento sobre la migración. Este trabajo muestra la insuficiencia de los modelos explicativos tradicionales y destaca que el estudio de la migración debe tener en cuenta simultáneamente la movilidad y la inmovilidad, las fuerzas centrífugas y centrípetas, las estructuras económicas y culturales y las dimensiones administrativas y políticas. 